\title{
Erratum
}

\section{Erratum to "Comparison of Oral Microbe Quantities from Tongue Samples and Subgingival Pockets"}

\author{
André Göhler $\mathbb{D}^{1,2}$ Stefanie Samietz, ${ }^{3}$ Carsten Oliver Schmidt $\mathbb{D}^{4},{ }^{4}$ Thomas Kocher $\mathbb{D},^{5}$ \\ Ivo Steinmetz, ${ }^{1,2}$ and Birte Holtfreter $\mathbb{C}^{5}$ \\ ${ }^{1}$ Friedrich Loeffler Institute of Medical Microbiology, University Medicine Greifswald, Greifswald, Germany \\ ${ }^{2}$ Institute for Hygiene, Microbiology and Environmental Medicine, Medical University of Graz, Graz, Austria \\ ${ }^{3}$ Department of Prosthodontics, Gerostomatology and Biomaterials, University Medicine Greifswald, Greifswald, Germany \\ ${ }^{4}$ SHIP-Clinical-Epidemiological Research, Institute for Community Medicine, University Medicine Greifswald, \\ Greifswald, Germany \\ ${ }^{5}$ Department of Restorative Dentistry, Periodontology, Endodontology, and Preventive and Pediatric Dentistry, \\ University Medicine Greifswald, Greifswald, Germany
}

Correspondence should be addressed to Birte Holtfreter; birte.holtfreter@uni-greifswald.de

Received 24 May 2018; Accepted 30 May 2018; Published 19 July 2018

Copyright ( 2018 André Göhler et al. This is an open access article distributed under the Creative Commons Attribution License, which permits unrestricted use, distribution, and reproduction in any medium, provided the original work is properly cited.

In the article titled "Comparison of Oral Microbe Quantities from Tongue Samples and Subgingival Pockets" [1], there was an error in the Acknowledgments, which should be corrected as follows.

\section{Acknowledgments}

SHIP is part of the Community Medicine Research net (CMR) of the University of Greifswald, Germany, which is funded by the Federal Ministry of Education and Research under Grant nos. ZZ9603, 01ZZ0103, and 01ZZ0701 and the Ministry of Cultural Affairs as well as the Social Ministry of the Federal State of Mecklenburg-West Pomerania (http:// www.community-medicine.de). Microbial analysis and André Göhler were supported by the Federal Ministry of Education and Research under Grant no. 03138010. The authors acknowledge support for the article processing charge from the DFG (German Research Foundation, 393148499) and the Open Access Publication Fund of the University of Greifswald. The authors acknowledge the SHIP data collection team, which comprises dental and medical examiners, technicians, interviewers, and assistants. The authors also thank Anne Kohler and Claudia Wiede for their technical assistance.

\section{References}

[1] A. Göhler, S. Samietz, C. O. Schmidt, T. Kocher, I. Steinmetz, and B. Holtfreter, "Comparison of oral microbe quantities from tongue samples and subgingival pockets," International Journal of Dentistry, vol. 2018, Article ID 2048390, 10 pages, 2018. 




Advances in
Public Health



Case Reports in

Medicine

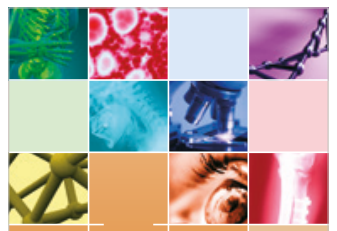

niernational Journal of

Biomaterials
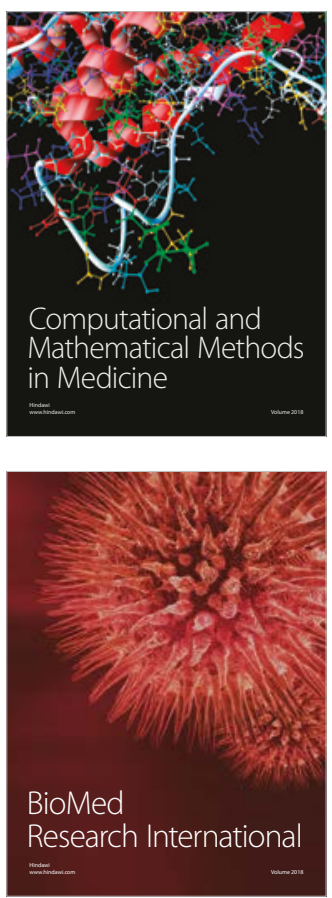

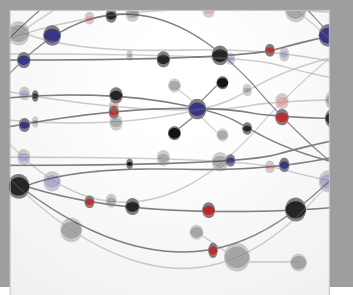

The Scientific World Journal Dentistry

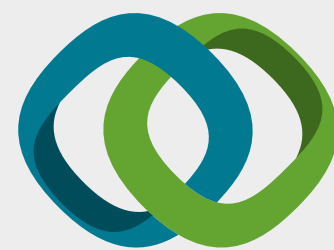

Hindawi

Submit your manuscripts at

www.hindawi.com
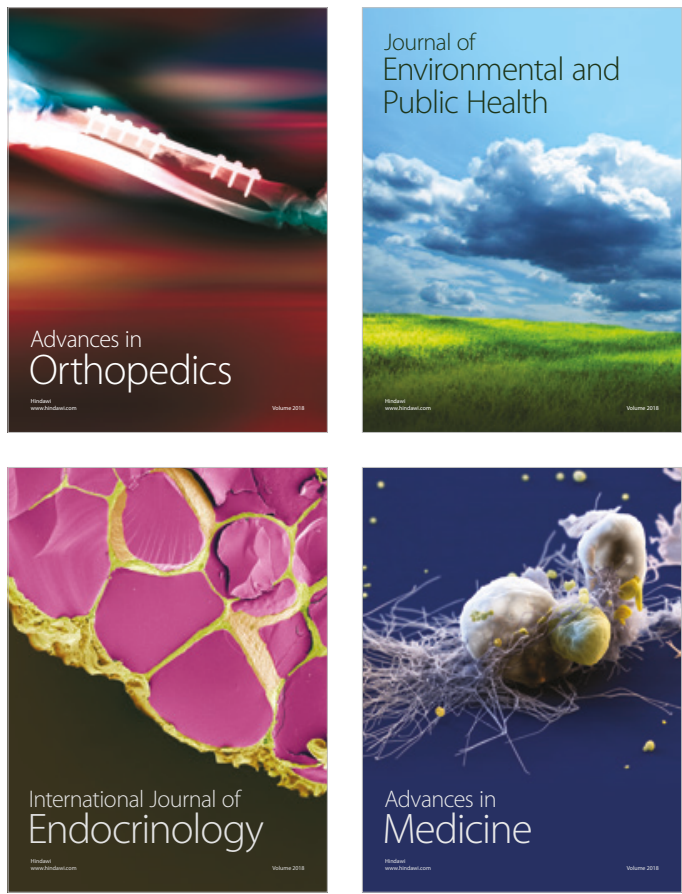


Scientifica



Case Reports in

Dentistry
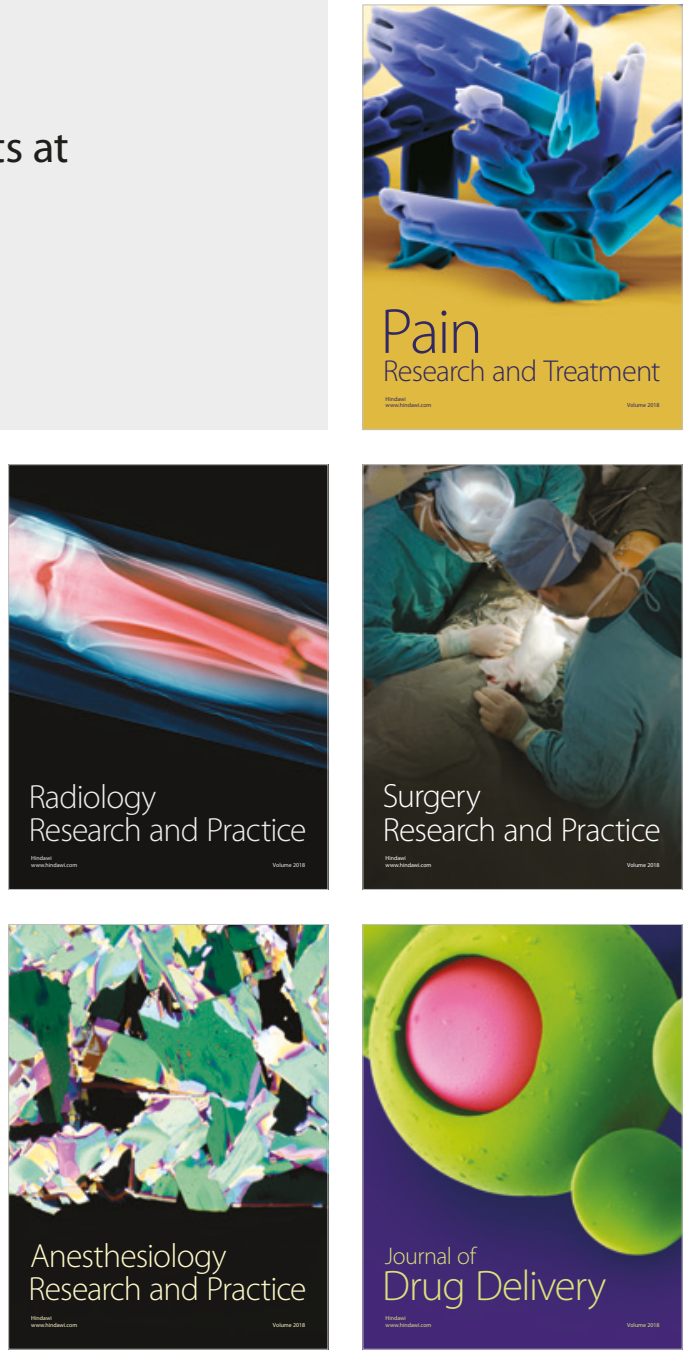